\title{
CONTROL LYAPUNOV FUNCTIONS FOR HOMOGENEOUS "JURDJEVIC-QUINN" SYSTEMS
}

\author{
LUDOVIC FAUBOURG $^{1}$ AND JEAN-BAPTISTE POMET ${ }^{1}$
}

\begin{abstract}
This paper presents a method to design explicit control Lyapunov functions for affine and homogeneous systems that satisfy the so-called "Jurdjevic-Quinn conditions". For these systems a positive definite function $V_{0}$ is known that can only be made non increasing by feedback. We describe how a control Lyapunov function can be obtained via a deformation of this "weak" Lyapunov function. Some examples are presented, and the linear quadratic situation is treated as an illustration.

Résumé. Cet article présente une méthode permettant de construire explicitement des fonctions de Lyapunov contrôlées pour des systèmes affines et homogènes vérifiant les conditions dites "de JurdjevicQuinn". Pour de tels systèmes, on connaît une fonction définie positive $V_{0}$ et une loi de commande qui fait seulement décroître $V_{0}$, avec une dérivée qui peut s'annuler en certains points. Nous montrons comment il est possible d'obtenir une fonction de Lyapunov contrôlée via une déformation de cette fonction de Lyapunov "au sens large". Quelques exemples sont présentés, et le cas linéaire quadratique est traité comme illustration.
\end{abstract}

AMS Subject Classification. 93D15, 93D30, 34D20.

Received July 1, 1999. Revised May 4, 2000.

\section{INTRODUCTION}

In all this paper we consider a control affine system:

$$
\dot{x}=f_{0}(x)+\sum_{k=1}^{m} u_{k} f_{k}(x),
$$

with state $x \in \mathbb{R}^{n}$ and control $\left(u_{1}, \ldots, u_{m}\right)=u \in \mathbb{R}^{m}$. The $f_{k}$ 's are smooth vector fields in $\mathbb{R}^{n}$. Smooth means of class $\mathrm{C}^{\infty}$.

For differentiable dynamical systems without control $\dot{x}=F(x)$, "Lyapunov's second theorem" states that existence of a positive definite and radially unbounded function that is decreasing along the trajectories of the system (such a function is called a Lyapunov function) implies asymptotic stability of its minimum. Asymptotic stability can therefore be proved by exhibiting such a function, this is the so-called "Lyapunov direct method" (see [12]). The scope of this method is general since the existence of a Lyapunov function is also necessary,

Keywords and phrases: Feedback stabilization, control Lyapunov function, Lyapunov design.

${ }^{1}$ INRIA, BP. 93, 06902 Sophia Antipolis, France;

e-mail: Ludovic.Faubourg@sophia.inria.fr \& Jean-Baptiste.Pomet@sophia.inria.fr

(c) EDP Sciences, SMAI 2000 
see [16] for a proof with a right hand side that is only assumed to be continuous. However an unfortunate drawback is that there is no systematic way to build explicitely this Lyapunov function.

For control systems, the "Lyapunov design" of a stabilizing control law (see [3]), based on Lyapunov direct method, consists in designing a positive definite and radially unbounded function together with a continuous feedback law that assigns this function to be a Lyapunov function for the closed-loop system. Artstein's theorem (see $[2,3,24])$ points out exactly which relations (control Lyapunov function, small control property, see below) a function has to satisfy in order to allow existence of a feedback law that makes it decrease.

Definition 1.1 (assignable Lyapunov functions). Let $V$ be a positive definite and radially unbounded function. We say that a continuous $u: \mathbb{R}^{n} \mapsto \mathbb{R}^{m}$ assigns $V$ to be a Lyapunov function for the closed loop system (1) if the derivative of $V$ along the trajectories of the closed loop system is negative definite, that means that for all $x$ in $\mathbb{R}^{n} \backslash\{0\}$ :

$$
L_{f_{0}} V(x)+\sum_{k=1}^{m} u_{k} L_{f_{k}} V(x)<0
$$

Theorem 1.2 (Artstein's theorem). Let $V$ be a positive definite and radially unbounded function. There exists a continuous feedback that assigns $V$ to be a Lyapunov function for the closed loop system (1) if and only if

(a) it is a control Lyapunov function (CLF):

$$
\left.\forall x \in \mathbb{R}^{n} \backslash\{0\} ; \quad \begin{array}{c}
L_{f_{1}} V(x)=0 \\
\vdots \\
L_{f_{m}} V(x)=0
\end{array}\right\} \Longrightarrow L_{f_{0}} V(x)<0 ;
$$

(b) it satisfies the small control property (SCP): for any $\epsilon>0$, there is a $\delta>0$ such that for all $x \in \mathbb{R}^{n} \backslash\{0\}$,

$$
\|x\|<\delta \Longrightarrow \exists u\left\{\begin{array}{l}
\|u\|<\epsilon \\
L_{f_{0}+\sum_{k=1}^{m} u_{k} f_{k}} V(x)<0 .
\end{array}\right.
$$

This is an existence result, but some explicit formulae have been given, like for instance:

Proposition 1.3 (Sontag's formula [24]). If the function $V$ is a control Lyapunov function that satisfies the small control property for the affine control system (1) then the control $u=\left(u_{1}, \ldots, u_{m}\right)$ defined by

$$
u_{k}=\left\{\begin{array}{lll}
-\frac{a+\sqrt{a^{2}+\left(\sum_{i=1}^{m} b_{i}{ }^{2}\right)^{2}}}{\sum_{i=1}^{m} b_{i}{ }^{2}} b_{k} & \text { if } \quad b_{k} \neq 0 \\
0 & \text { if } \quad b_{k}=0
\end{array}\right.
$$

where $a=L_{f_{0}} V(x)$ and $b_{k}=L_{f_{k}} V(x)$ for all $k$ in $\{1 \ldots m\}$, is a continuous and asymptotically stabilizing feedback law. Besides it assigns $V$ to be a Lyapunov function.

In this paper we will not refer to this general expression because we will restrict our attention to homogeneous systems. In this particular case a simpler construction can be made (see Prop. 2.5 below) that moreover keeps the homogeneity of the closed loop system.

Many others methods than building a control Lyapunov function exists in the literature to design stabilizing feedback law. However, it is well known that a control Lyapunov function, when it is available, is a convenient tool for many reasons: for instance, the negative definite function $\dot{V}$ allows one to quantify robustness to model errors or perturbations in the control (see [7], Sect. 3.3). Also, it can be used to perform Lyapunov redesign to enhance robustness properties (see [15], Sect. 5.5). Finally, some simple backstepping-like techniques for adding an integrator require a strict Lyapunov function (see [7]). 
For these reasons it is interesting to obtain control Lyapunov functions for systems that may be asymptotically stabilized by other methods. Of course, the converse Lyapunov theorems (see for instance $[12,16]$ ) imply the existence of functions that are strictly decreasing along the solutions of the closed loop systems and finally control Lyapunov functions that are assigned by the same control law. However these theorems are far from giving an explicit construction of these functions. This work is an attempt to give an explicit design of control Lyapunov functions as precise as possible when asymptotic stabilization can be solved via the so called "Jurdjevic-Quinn method", also called "damping control". A preliminary version was presented in [9].

Note that for linear systems, the above mentioned converse Lyapunov theorems can be made constructive, and yield a quadratic Lyapunov function, see Section 6 for a discussion.

To our knowledge, systematically trying to build a control Lyapunov function in theses cases is a novel idea. Let us however mention two occurences in the litterature where such a step was taken in more particular cases, and as part of a proof in [18] (proof of Lem. 2) for linear systems with saturations and in [19] (proof of Cor. 2.16) for cascaded systems with some growth assumptions, control Lyapunov functions have been obtained based on a quadratic Lyapunov function for the linear approximation. See Remark 3.4 for more details on these references. The present paper deals with more general affine control systems, although these results are not strico sensu a consequence of ours since we assume homogeneity.

The paper is organized as follows. Section 2 is devoted to introduce some classical notations and properties about differential geometry and homogeneity. After making precise the class of systems we deal with in Section 3 , the result consisting in reshaping a Lyapunov function in the direction of a vector field is presented in Section 4. After some comments on the result in Section 5, we pay attention to three examples in the last sections: the linear case is studied in Section 6 as a particular situation of the results, in Section 7 the problem of stabilization of an underactuated rigid body is considered and in Section 8 we deal with the so-called TORA system. Although this last system does not satisfy all the hypotheses of our results, the method leads to a control Lyapunov function for this system.

\section{Some notations AND PROPERTIES ABOUt VECTOR FIELDS AND HOMOGENEITY}

Let us precise our notations and recall basic properties. For an entire overview, the reader can refer to $[14]$ and $[25]$.

Definition 2.1 (Flow of a vector field). Let $X$ be a vector field on $\mathbb{R}^{n}$. We define $\phi_{X}^{t}(x)$ by:

$$
\begin{aligned}
\frac{\partial}{\partial t}\left(\phi_{X}^{t}(x)\right) & =X\left(\phi_{X}^{t}(x)\right) \\
\phi_{X}^{0}(x) & =x
\end{aligned}
$$

In general, $(t, x) \mapsto \phi_{X}^{t}(x)$ is defined on a neighborhood of $\{t=0\}$ in $\mathbb{R}^{n+1}$. If it is defined for all $(t, x)$ in $\mathbb{R}^{n+1}$, the vector field $X$ is called complete. For a fixed choice of $x, t \mapsto \phi_{X}^{t}(x)$ is a solution of $\dot{x}=X(x)$. For a fixed $t, x \mapsto \phi_{X}^{t}(x)$ is called the flow of $X$ at time $t$, it is a diffeomorphism from an open set of $\mathbb{R}^{n}$ onto its image (from $\mathbb{R}^{n}$ onto $\mathbb{R}^{n}$ if $X$ is complete).

For any function $h$, we denote by $L_{X} h$ its Lie derivative along $X$ :

$$
L_{X} h(x)=\left.\frac{d}{d t}\right|_{t=0} h\left(\phi_{X}^{t}(x)\right)
$$

Proposition 2.2. Let $X$ be a vector field on $\mathbb{R}^{n}$ and $\varphi$ a diffeomorphism from $\mathbb{R}^{n}$ onto $\mathbb{R}^{n}$. Then for all $t$, $\varphi \circ \phi_{X}^{t} \circ \varphi^{-1}$ is the flow at time $t$ of a vector field denoted by $\varphi * X$ and called the conjugate of $X$ by $\varphi$ :

$$
\phi_{\varphi * X}^{t}=\varphi \circ \phi_{X}^{t} \circ \varphi^{-1} .
$$


In coordinates, the expression of $\varphi * X$ is given by:

$$
\varphi * X(y)=\frac{\partial \varphi}{\partial x}\left(\varphi^{-1}(y)\right) X\left(\varphi^{-1}(y)\right)
$$

In the sequel we will also use the notion of homogeneity. Let us define precisely what we mean by homogeneous function and homogeneous vector field.

Definition 2.3. For a fixed choice of coordinates on $\mathbb{R}^{n}$, and for $n$ positive real numbers $r_{1}, \ldots, r_{n}$,

(a) a dilation of weight $r=\left(r_{1}, \ldots, r_{n}\right)$ is a family of maps $\left(\delta_{\mu}^{r}\right)$ defined, for all $\mu>0$ and for all $x$ in $\mathbb{R}^{n}$, by:

$$
\delta_{\mu}^{r}(x)=\left(\mu^{r_{1}} x_{1}, \ldots, \mu^{r_{n}} x_{n}\right)
$$

(b) a function $V: \mathbb{R}^{n} \mapsto \mathbb{R}$ is homogeneous of degree $\tau$ with respect to the family of dilations $\left(\delta_{\mu}^{r}\right)$ if and only if for all $\mu>0$,

$$
V \circ \delta_{\mu}^{r}=\mu^{\tau} V
$$

(c) a vector field $X$ is homogeneous of degree $\sigma$ with respect to the family of dilations $\left(\delta_{\mu}^{r}\right)$ if and only if for all $\mu>0$,

$$
\delta_{\mu}^{r} * X=\mu^{\sigma} X
$$

In the coordinates of the dilation, $X$ can be written $X=\sum f_{i} \frac{\partial}{\partial x_{i}}$, and $X$ is homogeneous of degree $\sigma$, if and only if, each $f_{i}$ is a homogeneous function of degree $\sigma+r_{i}$.

(d) In the sequel, we use the notation $\|\cdot\|_{\delta^{r}}$ for the homogeneous norm defined on $\mathbb{R}^{n}$ by

$$
\|x\|_{\delta^{r}}=\left(\sum_{j=1}^{n}\left|x_{j}\right|^{\frac{p}{r_{j}}}\right)^{\frac{1}{p}}
$$

for $p$ large enough that this is a smooth function of $x$ outside the origin. This homogeneous norm is obviously positive definite and homogeneous of degree 1 with respect to the family of dilations $\left(\delta_{\mu}^{r}\right)$. We might choose any function having these three properties.

In the sequel $r$ and the family of dilations $\left(\delta_{\mu}^{r}\right)$ are fixed. "Homogeneous of degree $d$ " will replace "homogeneous of degree $d$ with respect to $\left(\delta_{\mu}^{r}\right)$ ".

Lemma 2.4. Let $G$ be a complete vector field on $\mathbb{R}^{n} . G$ is homogeneous of degree 0 if and only if the flow of $G$ commutes with the family of dilations, i.e. for all $t$ in $\mathbb{R}$ and all positive $\mu$,

$$
\delta_{\mu}^{r} \circ \phi_{G}^{t}=\phi_{G}^{t} \circ \delta_{\mu}^{r}
$$

Proof. From (7) we have for all positive $\mu$ and all $t$ in $\mathbb{R}$ :

$$
\delta_{\mu}^{r} \circ \phi_{G}^{t} \circ\left(\delta_{\mu}^{r}\right)^{-1}=\phi_{\delta_{\mu}^{r} * G}^{t}
$$

Hence, from (9), $G$ is homogeneous of degree 0 if and only if $\delta_{\mu}^{r} \circ \phi_{G}^{t} \circ\left(\delta_{\mu}^{r}\right)^{-1}=\phi_{G}^{t}$ i.e. if and only if $\phi_{G}^{t}$ and $\delta_{\mu}^{r}$ commute. 
When all the vector fields $f_{i}$ of the system (1) are homogeneous, and when a homogeneous control Lyapunov function $V$ is known for this system, the following result, proved in [26] (see also the textbook [3] for the single-input case), gives a feedback control, simpler than Sontag's one (see Prop. 1.3), that assigns $V$ to be a Lyapunov function for the closed loop system. Moreover, this feedback law has the good property to keep the homogeneity of the closed loop system, that means that the right-hand side of the closed-loop system $\dot{x}=f_{0}(x)+\sum_{1}^{m} u_{k}(x) f_{k}(x)$, is homogeneous of the same degree as $f_{0}$.

Proposition 2.5. Consider the affine control system (1). Assume that each $f_{k}(k=0 \ldots m)$ is a vector field homogeneous of degree $c_{k}$, with $c_{0} \geq 0$ and $c_{k}<c_{0}(k=1 \ldots m)$. Assume as well that $V$ is a control Lyapunov function for the system, homogeneous of degree $d$. Then the feedback control, defined for all $x$ in $\mathbb{R}^{n}$ by:

$$
u_{k}(x)=-\alpha L_{f_{k}} V(x)\|x\|_{\delta^{r}}^{c_{0}-2 c_{k}-d}
$$

is homogeneous of degree $c_{0}-c_{k}(k=1 \ldots m)$. This makes the right-hand side of the closed-loop system $\dot{x}=f_{0}(x)+\sum_{k=1}^{m} u_{k}(x) f_{k}(x)$ homogeneous of degree $c_{0}$, and assigns the control Lyapunov function $V$ to be strictly decreasing if, $T$ being the set $\left\{x,\|x\|_{\delta^{r}}=1, L_{f_{0}} V(x) \geq 0\right\}$,

$$
\alpha>\sup _{x \in T} \frac{L_{f_{0}} V(x)}{\sum_{k=1}^{m}\left(L_{f_{k}} V(x)\right)^{2}\|x\|_{\delta^{r}}^{c_{0}-2 c_{k}-d}} .
$$

\section{Problem statement}

In [13], Jurdjevic and Quinn noticed that a first integral of the drift vector field plus some controllability conditions allow one to derive smooth asymptotically stabilizing control laws. This method has been next generalized in many papers, as $[3,11,21]$. In this paper we call "Jurdjevic-Quinn type" systems, these that allow the use of this stabilization method.

Let us give the specific assumption we need here. Consider the affine control system (1). For a function $V_{0}$ and for an integer $L$, define the set $\mathcal{W}_{L}\left(V_{0}\right)$ to be:

$$
\mathcal{W}_{L}\left(V_{0}\right)=\left\{\begin{array}{c}
x \in \mathbb{R}^{n}, L_{f_{0}} V_{0}(x)=L_{a d_{f_{0}}^{i}\left(f_{k}\right)} V_{0}(x)=0 \\
k=1 \ldots m ; i=0 \ldots L
\end{array}\right\}
$$

Note that this set decreases as $L$ increases.

Assumption 3.1 (Jurdjevic-Quinn conditions). We assume that a function $V_{0}: \mathbb{R}^{n} \rightarrow \mathbb{R}$ is known and has the following three properties: it is smooth, positive definite and radially unbounded; it satisfies:

$$
\forall x \in \mathbb{R}^{n}, L_{f_{0}} V_{0}(x) \leq 0 ;
$$

it is such that there is an integer $L$ such that

$$
\mathcal{W}_{L}\left(V_{0}\right) \text { is reduced to }\{0\} \text {. }
$$

Remark 3.2. For many control systems (see the examples in Sects. 7 and 8), the natural drift vector field does not meet (14), but a preliminary feedback, by adding to it a linear combination of the control vector fields, gives an $f_{0}$ satisfying (14). Further comments on the consequence of this preliminary feedback are given in Section 5 .

Following $[3,11,13,21]$, one may set:

$$
u_{k}(x)=-L_{f_{k}} V_{0}(x) \quad(k=1, \ldots, m) .
$$


The derivative of $V_{0}$ along the closed-loop system is then given by,

$$
\dot{V}_{0}=L_{f_{0}} V_{0}-\sum_{k=1}^{m}\left(L_{f_{k}} V_{0}\right)^{2} .
$$

This equation shows that $\dot{V}_{0}$ is non positive and vanishes at points where the $L_{f_{k}} V_{0}(k=0 \ldots m)$ vanish. If such points exist, condition (15) together with LaSalle Invariance principle (see $[12,17]$ ) allow one to conclude to asymptotic stability of the origin:

Proposition 3.3. If system (1) satisfies Assumption 3.1, then formula (16) defines a continuous asymptotically stabilizing feedback control law.

However, $V_{0}$ is clearly not a control Lyapunov function, because there are in general some points where all the $L_{f_{k}} V_{0}(k=0 \ldots m)$ vanish. In the sequel, we "re-shape" this function to build a control Lyapunov function.

Remark 3.4. In [18] (proof of Lem. 2), the case where $f_{0}$ is a linear vector field and where the control vector fields depends on the control (systems are non affine in the control but it has a particular structure) is studied. A positive definite and radially unbounded function together with a feedback law that assigns it to be a strict Lyapunov function is obtained following the idea of [19] (proof of Cor. 2.16). This explicit construction consists in finding a Lyapunov function for the linear approximation (via Lyapunov equation for instance, see Sect. 6, Eq. (34)), and making a combination of this function and the above defined $V_{0}$. It is not clear how this approach might be generalized. We do not follow this track here.

Remark 3.5. Let us clarify the status of our assumptions with respect to the literature (and with the help of [22]). Denoting by $\mathcal{L}$ the union of all the trajectories of $\dot{x}=f_{0}(x)$ that remain in the set $\left\{x \in \mathbb{R}^{n}, L_{f_{k}} V_{0}(x)=\right.$ $0, k=0, \ldots, m\}$, LaSalle's invariance principle states that $\mathcal{L}=\{0\}$ is a necessary and sufficient conditions for (16) to be an asymptotically stabilizing control law. All the assumptions made in the literature may be viewed as sufficient conditions for $\mathcal{L}=\{0\}$.

In the early works [11,13], some simpler and more restrictive assumptions were made instead of Assumption 3.1: (14) was replaced by $L_{f_{0}} V_{0} \equiv 0$ ( $V_{0}$ is a first integral of the drift vector field) and (15) was replaced by the "ad-condition" $\left(r k\left\{a d_{f_{0}}^{i} f_{k}, \quad 1 \leq k \leq m, i \geq 0\right\}=n\right)$ plus the origin being the only critical point of $V_{0}$. These assumptions are rather restrictive, but they have the advantage of "decoupling" the properties of the control vector fields and these of $f_{0}$.

There has then been an effort to weaken these assumptions. By differentiation along the trajectories, it is clear that the set

$$
\mathcal{W}_{\text {gen }}=\left\{\begin{array}{c}
x \in \mathbb{R}^{n}, L_{f_{0}}^{i+1} V_{0}(x)=L_{f_{0}}^{i} L_{f_{k}} V_{0}(x)=0 \\
k=1 \ldots m ; i \in \mathbb{N}
\end{array}\right\}
$$

contains $\mathcal{L}$, defined above, and in fact they coincide for real analytic vector fields. In [21] the stabilization result is stated under the condition $\mathcal{W}_{\text {gen }}=\{0\}$. It is noted there that, with

$$
\mathcal{W}_{\text {gen }}^{\prime}=\left\{\begin{array}{c}
x \in \mathbb{R}^{n}, L_{f_{0}}^{i+1} V_{0}(x)=L_{a d_{f_{0}}\left(f_{k}\right)} V_{0}(x)=0 \\
k=1 \ldots m ; i \in \mathbb{N}
\end{array}\right\},
$$

the condition $\mathcal{W}_{\text {gen }}=\{0\}$ is equivalent to $\mathcal{W}_{\text {gen }}^{\prime}=\{0\}$. Note that in general (without further assumption like $L_{f_{0}} V_{0}$ identically zero) $L_{a d_{f_{0}}^{i}\left(f_{k}\right)} V_{0}(x)=L_{f_{0}}^{i} L_{f_{k}} V_{0}(x)$ is true only for $x$ in $\mathcal{L}$, so that the sets $\mathcal{W}_{\text {gen }}$ and $\mathcal{W}_{\text {gen }}^{\prime}$ might not coincide if they are not reduced to zero.

The set $\mathcal{W}_{L}\left(V_{0}\right)$ contains obviously $\mathcal{W}_{\text {gen }}^{\prime}$ and so our condition is stronger. However, in the very common situation where the drift vector field is conservative $\left(L_{f_{0}} V_{0} \equiv 0\right)$, the knowledge of the integer $L$ is our only restriction. 


\section{RESHAPING LyAPUNOV FUNCTIONS via THE FLOW OF A VECTOR FIELD}

For a complete vector field $G$, we define a function $V_{\lambda}$ from $V_{0}$ as follows:

$$
V_{\lambda}=V_{0} \circ \phi_{G}^{\lambda}
$$

where $\phi_{G}^{\lambda}$ is the flow of the vector field $G$, see (5). We shall always consider a vector field $G$ that is smooth except possibly at $x=0$, with $G(0)=0$.

In general, it is impossible to give an explicit expression of the flow $\phi_{G}^{\lambda}$ with some usual functions. For the purpose of making our method really constructive, we may use for small $\lambda$ (all our results will anyway be valid for small $\lambda$ only), a function $W_{\lambda}$ that is easier to compute and agrees with $V_{\lambda}$ up to order 1 with respect to $\lambda$ :

$$
W_{\lambda}(x)=V_{\lambda}(x)+\lambda^{2} a(\lambda, x),
$$

where $a$ is a smooth function on $[0,+\infty) \times \mathbb{R}^{n}$ that satisfies for all positive $\lambda, a(\lambda, 0)=0$. From Hadamard's Lemma, this property is equivalent to:

$$
\begin{array}{llll}
W_{\lambda}(0) & =V_{\lambda}(0) & & \forall \lambda \in[0,+\infty) \\
W_{0}(x) & =V_{0}(x) & & \forall x \in \mathbb{R}^{n} \\
\left.\frac{\partial W_{\lambda}(x)}{\partial \lambda}\right|_{\lambda=0} & =\left.\frac{\partial V_{\lambda}(x)}{\partial \lambda}\right|_{\lambda=0} & & \forall x \in \mathbb{R}^{n} .
\end{array}
$$

For instance the following $W_{\lambda}$ is of this type:

$$
W_{\lambda}(x)=V_{0}(x+\lambda G(x)) .
$$

Note that this formula is explicit (does not involve solving any differential or algebraic equation) and that it depends on the chosen coordinates whereas the definition of $V_{\lambda}$ in (17) is coordinate-free.

The function $V_{\lambda}$ is identically equal to $V_{0}$ when $\lambda$ is equal to zero. Since $L_{f_{0}} V_{0}$ vanishes at some points where $L_{f_{k}} V_{0}(k=1 \ldots m)$ vanish, and we want $L_{f_{0}} V_{\lambda}$ to be negative at points where $L_{f_{k}} V_{\lambda}(k=1 \ldots m)$ vanish, it is natural to require that $L_{f_{0}} V_{\lambda}$ be a decreasing function of $\lambda$ for small values of $\lambda$ and at points where $L_{f_{k}} V_{0}(k=0 \ldots m)$ vanish. We require the following slightly stronger property:

$$
\left.\forall x \in \mathbb{R}^{n} \backslash\{0\}, \begin{array}{c}
L_{f_{0}} V_{0}(x)=0 \\
L_{f_{1}} V_{0}(x)=0 \\
\vdots \\
L_{f_{m}} V_{0}(x)=0
\end{array}\right\}\left.\Longrightarrow \frac{d}{d \lambda}\right|_{\lambda=0}\left(L_{f_{0}} V_{\lambda}(x)\right)<0 .
$$

The same discussion holds replacing $V_{\lambda}$ with $W_{\lambda}$ satisfying (18).

Reshaping $V_{0}$ via the flow of a vector field $G$ has the interesting advantage that it replaces the above difficult condition on $V_{\lambda}$ given by equation (20) by a simpler condition on $G$ as shown by the following lemma:

Lemma 4.1. For function $V_{\lambda}$ and $W_{\lambda}$ defined by (17) and (18), we have for all $x \in \mathbb{R}$,

$$
\left.\frac{d}{d \lambda}\right|_{\lambda=0}\left(L_{f_{0}} V_{\lambda}\right)(x)=\left.\frac{d}{d \lambda}\right|_{\lambda=0}\left(L_{f_{0}} W_{\lambda}\right)(x)=L_{f_{0}} L_{G} V_{0}(x) .
$$

Proof. Let the function $\chi$ be defined by $\chi(t, \lambda, x)=V_{0}\left(\phi_{G}^{\lambda}\left(\phi_{f_{0}}^{t}(x)\right)\right)$. From (6) and the definition (17) of $V_{\lambda}$, the left-hand side of (21) is equal to $\frac{\partial^{2} \chi}{\partial \lambda \partial t}(0,0, x)$. From (18), the expression with $W_{\lambda}$ instead of $V_{\lambda}$ is also equal to $\frac{\partial^{2} \chi}{\partial \lambda \partial t}(0,0, x)$. On the other hand, applying (6) twice (with $X=G$ and $X=f_{0}$ ), the right-hand side is equal to $\frac{\partial^{2} \chi}{\partial t \partial \lambda}(0,0, x)$. This proves $(21)$ because the partial derivatives commute. 
Unfortunately, we are not able to prove that condition (20) is sufficient, in general, for $V_{\lambda}$ to be a control Lyapunov function $^{1}$. This is however true for control affine systems which have some homogeneity properties:

Theorem 4.2. Assume that the control affine system (1) meets the Jurdjevic-Quinn conditions (Assumpt. 3.1) with a function $V_{0}$ that is homogeneous of degree $d$. Besides, assume that each $f_{k}(k=0 \ldots m)$ is homogeneous of degree $c_{k}$ with $c_{k} \geq 0$ and $c_{k}<c_{0}(k=1 \ldots m)$.

Let $G$ be an homogeneous vector field of degree 0 which satisfies, for all $x$ in $\mathbb{R}^{n} \backslash\{0\}$,

$$
\left.\begin{array}{c}
L_{f_{0}} V_{0}(x)=0 \\
L_{f_{1}} V_{0}(x)=0 \\
\vdots \\
L_{f_{m}} V_{0}(x)=0
\end{array}\right\} \Longrightarrow L_{f_{0}} L_{G} V_{0}(x)<0 .
$$

Then there exists a positive real $\lambda_{0}$ such that for all $\lambda$ satisfying $0<\lambda<\lambda_{0}, V_{\lambda}$ is a homogeneous control Lyapunov function of degree $d$. The same property is satisfied by $W_{\lambda}$ given by (19) in the coordinates of the dilation. Any $W_{\lambda}$ that agrees with (18) also satisfies this property provided that it is homogeneous and definite positive.

Of course, in order to give an explicit method to build a control Lyapunov function, the previous result is not sufficient: one still needs to find a vector field $G$ satisfying (22). The following result gives a "universal" way of designing such a vector field. Note that the above homogeneity requirement on $G$ makes it difficult to construct such a vector field smooth at the origin.

It is natural to write $G$ as a linear combination of some $a d_{f_{0}}^{i} f_{k}$, and translate (22) into equations for the coefficients, $\lambda_{i, k}$, of this combination:

$$
G=\sum_{k=1}^{m} \sum_{i=0}^{I_{k}} \lambda_{i, k} a d_{f_{0}}^{i} f_{k}
$$

Then $\left[f_{0}, G\right]=\sum \sum\left(L_{f_{0}} \lambda_{i, k} a d_{f_{0}}^{i} f_{k}+\lambda_{i, k} a d_{f_{0}}^{i+1} f_{k}\right)$. So we have,

$$
\begin{aligned}
L_{\left[f_{0}, G\right]} V_{0} & =\sum_{k=1}^{m} \sum_{i=0}^{I_{k}}\left(L_{f_{0}} \lambda_{i, k} L_{a d_{f_{0}}^{i} f_{k}} V_{0}+\lambda_{i, k} L_{a d_{f_{0}}^{i+1} f_{k}} V_{0}\right) \\
& =\sum_{k=1}^{m} L_{f_{0}} \lambda_{0, k} L_{f_{k}} V_{0}+\sum_{k=1}^{m} \lambda_{I_{k}, k} L_{a d_{f_{0}}^{I_{k}+1} f_{k}}+\sum_{k=1}^{m} \sum_{i=1}^{I_{k}-1}\left(L_{f_{0}} \lambda_{i+1, k}+\lambda_{i, k}\right) L_{a d_{f_{0}}^{i+1} f_{k}} V_{0}
\end{aligned}
$$

At points where the left hand of (22) is true, the first sum vanishes and $L_{\left[f_{0}, G\right]} V_{0}(x)=L_{f_{0}} L_{G} V_{0}(x)$ (see the proof of Th. 4.3 for details on this point). As developed in Remark 5.3, it may be a good idea on a concrete example, to start from these considerations to design some functions $\lambda_{i, k}$. The following result gives a "universal" way of designing the $\lambda_{i, k}$ so that vector field $G$ meets condition (22). All $I_{k}$ is taken equal to $L-1$, with $L$ coming from (15) in Assumption 3.1.

Theorem 4.3. Assume that the control affine system (1) meets the Jurdjevic-Quinn conditions (Assumpt. 3.1) with a function $V_{0}$ that is homogeneous of degree $d$. Besides, assume that each $f_{k}(k=0 \ldots m)$ is homogeneous of degree $c_{k}$ with $c_{k} \geq 0$ and $c_{k}<c_{0}(k=1 \ldots m)$.

\footnotetext{
${ }^{1}$ What is true in general (i.e. without the homogeneity assumption) is that $V_{\lambda}$ satisfies, for $\lambda>0$ small enough, the condition (2) to be a control Lyapunov function, but only in an annulus, more precisely in a compact region excluding the origin (since the origin is excluded (3) does not make sense). Such a function does not allow one to design an asymptotically stabilising feedback law since nothing can be granted in a neighborhood of the origin. It however obviously makes it possible to design a feedback law that makes the origin practically stable, see [8]. This can be proved using part of the proof of Theorem 4.2 since what is proved on the unit sphere $S$ could be proved on any compact set that does not contain the origin.
} 
Then the vector field $G$ defined by (26) and (27) is homogeneous of degree 0 and satisfies the condition (22):

$$
G=\sum_{i=0}^{L-1} \sum_{k=1}^{m} \lambda_{i, k} a d_{f_{0}}^{i} f_{k},
$$

with $\lambda_{i, k}(i=0, \ldots, L-1 ; k=1, \ldots, m)$ some functions defined by:

$$
\left\{\begin{aligned}
\lambda_{i, k} & =\sum_{j=i}^{L-1}(-1)^{j-i+1} \frac{L_{a d_{f_{0}}^{(2 j-i+1)}\left(f_{k}\right)} V_{0}}{\left(V_{0}\right)^{\alpha_{j, k}}} \\
\alpha_{j, k} & =\frac{(2 j+1) c_{0}+2 c_{k}+d}{d}
\end{aligned}\right.
$$

The following lemma is used in the proof of Theorem 4.2, but has some interest in itself.

Lemma 4.4. If $V_{0}$ is homogeneous of degree $d$ and the vector field $G$ is homogeneous of degree zero then, for all $\lambda \geq 0$, the functions $V_{\lambda}$ defined by (17) and $W_{\lambda}$ defined by (19) are homogeneous of degree $d$.

Of course, for a general $W_{\lambda}$ satisfying (18), homogeneity is not granted except if, for $\lambda \geq 0, x \mapsto a(\lambda, x)$ is homogeneous of the same degree as $V_{0}$.

Proof. Since $G$ is homogeneous of degree 0, Lemma 2.4 implies that $\delta_{\mu}^{r}$ commutes with the flow of the vector field $G$ at any positive time $\lambda$. Then for all $\mu>0$ and $\lambda \geq 0$,

$$
V_{\lambda} \circ \delta_{\mu}^{r}=V_{0} \circ \phi_{G}^{\lambda} \circ \delta_{\mu}^{r}=V_{0} \circ \delta_{\mu}^{r} \circ \phi_{G}^{\lambda}=\mu^{d} V_{0} \circ \phi_{G}^{\lambda}=\mu^{d} V_{\lambda}
$$

This precisely means that $V_{\lambda}$ is homogeneous of degree $d$. The function $W_{\lambda}=V_{0} \circ(I d+\lambda G)$ is also homogeneous of degree $d$ because:

$$
W_{\lambda} \circ \delta_{\mu}^{r}=V_{0} \circ\left(\delta_{\mu}^{r}+\lambda G \circ \delta_{\mu}^{r}\right)=V_{0} \circ \delta_{\mu}^{r} \circ(I d+\lambda G)=\mu^{d} V_{0} \circ(I d+\lambda G) .
$$

This ends the proof of the lemma.

Proof of Theorem 4.2. We are going to prove that $V_{\lambda}$ is a control Lyapunov function. The proof that $W_{\lambda}$ satisfies the same property follows exactly along the same lines because only the properties of Lemmas 4.1 and 4.4 are used.

From Lemma 4.4 the functions $V_{\lambda}$ are homogeneous of degree $d$. Since each vector field $f_{k}(k=0 \ldots m)$ is homogeneous of degree $c_{k}$, each function $L_{f_{k}} V_{\lambda}(k=0 \ldots m)$ is homogeneous of degree $c_{k}+d$. Let

$$
S=\left\{y \in \mathbb{R}^{n},\|y\|_{\delta^{r}}=1\right\}
$$

be the homogeneous unit sphere. For any $x \in \mathbb{R}^{n} \backslash\{0\}$, there exists $y \in S$ and a real number $\mu>0$ such that $x=\delta_{\mu}(y)$. Consequently, the homogeneity of each $L_{f_{k}} V_{\lambda}$ implies that it is equivalent to prove that for all $x \in \mathbb{R}^{n}, L_{f_{0}} V_{\lambda}(x)$ is negative when each $L_{f_{k}} V_{\lambda}(x)(k=1 \ldots m)$ vanishes and to prove this property only for $x$ in $S$.

To simplify the proof of this fact, let us introduce some notations:

$$
\begin{aligned}
E & =\left\{x \in S, L_{f_{0}} L_{G} V_{0}(x) \geq 0\right\}, \\
F & =\left\{x \in S, L_{f_{0}} V_{0}(x)=0\right\} \\
\Gamma & =\left\{x \in S, L_{f_{1}} V_{0}(x)=\ldots=L_{f_{m}} V_{0}(x)=0\right\}, \\
\Gamma_{\lambda} & =\left\{x \in S, L_{f_{1}} V_{\lambda}(x)=\ldots=L_{f_{m}} V_{\lambda}(x)=0\right\}, \\
\Gamma^{\prime} & =\Gamma \cap F=\left\{x \in S, L_{f_{0}} V_{0}(x)=L_{f_{1}} V_{0}(x)=\ldots=L_{f_{m}} V_{0}(x)=0\right\} .
\end{aligned}
$$


With these notations, (22) is equivalent to

$$
\Gamma^{\prime} \cap E=\emptyset
$$

and from the above considerations, $V_{\lambda}$ is a control Lyapunov function if and only if $L_{f_{0}} V_{\lambda}$ is negative on the set $\Gamma_{\lambda}$.

Compactness of $S$ and continuity of $(x, \lambda) \mapsto L_{f_{k}} V_{\lambda}$ imply that any neighborhood of $\Gamma$ in $S$, say for instance

$$
N_{q}(\Gamma)=\{x \in S, d(x, \Gamma) \leq q\}, q>0,
$$

contains the sets $\Gamma_{\lambda}$, for $\lambda$ small enough (if not, there would exist a sequence outside that neighborhood that would converge to a point in $\Gamma)$. More precisely for any $q>0$ there exists $\lambda_{2}(q)>0$ s.t,

$$
0<\lambda<\lambda_{2}(q), \Rightarrow \Gamma_{\lambda} \subset N_{q}(\Gamma)
$$

To end the proof, it remains to prove that there exists two positive real numbers $\lambda_{0}$ and $q$ such that,

$$
\left.\begin{array}{r}
0<\lambda<\lambda_{0} \\
x \in N_{q}(\Gamma)
\end{array}\right\} \Longrightarrow L_{f_{0}} V_{\lambda}(x)<0
$$

First, note that from (28), $\Gamma^{\prime}$ and $E$ are two disjoint compact sets, so that the distance between them is strictly positive. Let $q^{\prime}$ be such that $0<q^{\prime}<d\left(E, \Gamma^{\prime}\right)$, and define:

$$
N_{q^{\prime}}\left(\Gamma^{\prime}\right)=\left\{x \in S, d\left(x, \Gamma^{\prime}\right) \leq q^{\prime}\right\}
$$

This set is compact and disjoint from $E$. Define the function $\chi: \mathbb{R} \times \mathbb{R}^{n} \rightarrow(-\infty, 0]$ by $\chi(\lambda, x)=L_{f_{0}} V_{\lambda}(x)$. It is smooth away from $x=0$, and in particular on $[0,+\infty) \times N_{q^{\prime}}\left(\Gamma^{\prime}\right)$. From Lemma $4.1, \frac{\partial \chi}{\partial \lambda}(0, x)=L_{f_{0}} L_{G} V_{0}(x)$ for all $x$, so that $\frac{\partial \chi}{\partial \lambda}(0, x)$ is strictly negative for $x$ in $N_{q^{\prime}}\left(\Gamma^{\prime}\right)$. Compactness of $N_{q^{\prime}}\left(\Gamma^{\prime}\right)$ implies that:

$$
\max _{x \in N_{q^{\prime}}\left(\Gamma^{\prime}\right)} \frac{\partial \chi}{\partial \lambda}(0, x)=-\beta<0 .
$$

Continuous differentiability of $\chi$ on $[0,+\infty) \times N_{q^{\prime}}\left(\Gamma^{\prime}\right)$ implies that, for a certain $\lambda_{1}>0$ :

$$
(\lambda, x) \in\left[0, \lambda_{1}\right] \times N_{q^{\prime}}\left(\Gamma^{\prime}\right) \Longrightarrow \frac{\partial \chi}{\partial \lambda}(\lambda, x)=-\frac{\beta}{2}<0 .
$$

By integrating with respect to $\lambda$ on the interval $\left[0, \lambda_{1}\right]$ and taking into account the fact that, from (14), $\chi(0, x) \leq 0$ for all $x$, one obtains that $\chi(\lambda, x)<-\frac{\beta \lambda}{2}$ for $(\lambda, x)$ in $\left[0, \lambda_{1}\right] \times N_{q^{\prime}}\left(\Gamma^{\prime}\right)$. This proves:

$$
\left.\begin{array}{c}
0<\lambda<\lambda_{1} \\
x \in N_{q^{\prime}}\left(\Gamma^{\prime}\right)
\end{array}\right\} \Longrightarrow L_{f_{0}} V_{\lambda}(x)<0
$$

Let us now introduce the set $\Gamma^{\prime \prime}=\Gamma \cap\left\{x \in S, d\left(x, \Gamma^{\prime}\right) \geq \frac{q^{\prime}}{2}\right\}$. If $\Gamma^{\prime \prime}$ is not empty, then, by their definition $\Gamma^{\prime \prime}$ and $F$ are two disjoint compact set, so that the distance between them is strictly positive. Pick $q^{\prime \prime}$ such that $0<q^{\prime \prime}<d\left(\Gamma^{\prime \prime}, F\right)$, and define

$$
N_{q^{\prime \prime}}\left(\Gamma^{\prime \prime}\right)=\left\{x \in S, d\left(x, \Gamma^{\prime \prime}\right) \leq q^{\prime \prime}\right\}
$$

The function $L_{f_{0}} V_{0}$ is strictly negative on this compact set. Hence it has a strictly negative maximum and again, by continuity of the above function $\chi$, we deduce that for a certain $\lambda_{2}>0$ :

$$
\left.\begin{array}{r}
0<\lambda<\lambda_{2} \\
x \in N_{q^{\prime \prime}}\left(\Gamma^{\prime \prime}\right)
\end{array}\right\} \Longrightarrow L_{f_{0}} V_{\lambda}(x)<0
$$


If $\Gamma^{\prime \prime}$ is empty then, setting $N_{q^{\prime \prime}}\left(\Gamma^{\prime \prime}\right)=\emptyset$ by convention, the above is trivially true for any $\lambda_{2}>0$ and $q^{\prime \prime}>0$.

Finally, let us define the two positive real numbers $q$ and $\lambda_{0}$ by $q=\min \left(\frac{q^{\prime}}{2}, q^{\prime \prime}\right)$ and $\lambda_{0}=\min \left(\lambda_{1}, \lambda_{2}\right)$. Let $\lambda$ be in $\left(0, \lambda_{0}\right)$ and $x$ be in $N_{q}(\Gamma)$. If $d\left(x, \Gamma^{\prime \prime}\right) \leq q^{\prime \prime}$ then $x$ belongs to $N_{q^{\prime \prime}}\left(\Gamma^{\prime \prime}\right)$. Since $\lambda_{0} \leq \lambda_{2}$, equation (32) proves that $L_{f_{0}} V_{\lambda}(x)<0$. If $d\left(x, \Gamma^{\prime \prime}\right)>q^{\prime \prime}$ we have $d(x, \Gamma)<d\left(x, \Gamma^{\prime \prime}\right)$; and, noticing that $\Gamma=\Gamma^{\prime \prime} \cup\{y \in$ $\left.\Gamma, d\left(y, \Gamma^{\prime}\right) \leq \frac{q^{\prime}}{2}\right\}$, we deduce that the points $y$ in $\Gamma$ such that $d(x, y)=d(x, \Gamma)$ satisfy $d\left(y, \Gamma^{\prime}\right) \leq \frac{q^{\prime}}{2}$. Then we have $d\left(x, \Gamma^{\prime}\right) \leq d(x, y)+d\left(y, \Gamma^{\prime}\right) \leq q^{\prime}$. Consequently $x$ is in $N_{q^{\prime}}\left(\Gamma^{\prime}\right)$. Since $\lambda_{0} \leq \lambda_{1}$, equation (31) proves that $L_{f_{0}} V_{\lambda}(x)<0$. This proves (30) and completes the proof of the theorem.

Proof of Theorem 4.3. To prove that $G$ given by (26) is homogeneous of degree 0 , it is enough, since each $a d_{f_{o}}^{i} f_{k}$ is a homogeneous vector field of degree $c_{k}+i c_{0}$, to prove that each $\lambda_{i, k}$ given by (27) is a homogeneous function of degree $-c_{k}-i c_{0}$. Indeed the $j^{\text {th }}$ term in the sum defining $\lambda_{i, k}$ has degree

$$
d+c_{k}+(2 j-i+1) c_{k}-\alpha_{j, k} d=-c_{k}-i c_{0} .
$$

Let us now prove that $G$ satisfies (22). If $G$ is given by (26), then we have already proved (see (25)) that

$$
L_{\left[f_{0}, G\right]} V_{0}(x)=\sum_{k=1}^{m} L_{f_{0}} \lambda_{0, k} L_{f_{k}} V_{0}+\sum_{k=1}^{m} \lambda_{L-1, k} L_{a d_{f_{0}}^{L} f_{k}} V_{0}+\sum_{i=1}^{L-2} \sum_{k=1}^{m}\left(L_{f_{0}} \lambda_{i+1, k}+\lambda_{i, k}\right) L_{a d_{f_{0}}^{i+1} f_{k}} V_{0} .
$$

Using (27), we have:

$$
\begin{aligned}
L_{f_{0}} \lambda_{i+1, k}+\lambda_{i, k}= & \sum_{j=i+1}^{L-1}(-1)^{j-i} L_{f_{0}} \frac{L_{a d_{f_{0}}^{(2 j-i)}\left(f_{k}\right)} V_{0}}{\left(V_{0}\right)^{\alpha_{j, k}}}+\sum_{j=i}^{L-1}(-1)^{j-i+1} \frac{L_{a d_{f_{0}}^{(2 j-i+1)}\left(f_{k}\right)} V_{0}}{\left(V_{0}\right)^{\alpha_{j, k}}} \\
= & \sum_{j=i+1}^{L-1}(-1)^{j-i} \frac{L_{f_{0}} L_{a d_{f_{0}}^{(2 j-i)}\left(f_{k}\right)} V_{0}-L_{a d_{f_{0}}^{(2 j-i+1)}\left(f_{k}\right)} V_{0}}{\left(V_{0}\right)^{\alpha_{j, k}}} \\
& -L_{f_{0}} V_{0}\left(\sum_{j=i+1}^{L-1}(-1)^{j-i} \alpha_{j, k} \frac{L_{a d_{f_{0}}^{(2 j-i)}\left(f_{k}\right)} V_{0}}{\left(V_{0}\right)^{\alpha_{j, k}+1}}\right)-\frac{L_{a d_{f_{0}}^{i+1} f_{k}} V_{0}}{\left(V_{0}\right)^{\alpha_{i, k}}} .
\end{aligned}
$$

Since $L_{f_{0}} V_{0}$ is non positive on $R^{n}$, it is maximum at all points where it vanishes. Consequently, for all $x$ such that $L_{f_{0}} V_{0}(x)=0$, we have, for any vector field $X, L_{X} L_{f_{0}} V_{0}(x)=0$, hence $L_{\left[f_{0}, X\right]} V_{0}(x)=L_{f_{0}} L_{X} V_{0}(x)$. Therefore at points where $L_{f_{0}} V_{0}$ vanishes, the first and the second term in the expression of $L_{f_{0}} \lambda_{i+1, k}+\lambda_{i, k}$ vanish, and $L_{\left[f_{0}, G\right]} V_{0}=L_{f_{0}} L_{G} V_{0}$, i.e.,

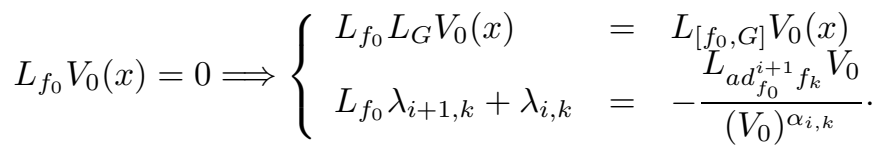

Since, from (27), $\lambda_{L-1, k}=-\frac{L_{a d_{f_{0}}^{L} f_{k}} V_{0}}{V_{0}{ }^{\alpha_{L-1, k}}}$, we have from the above calculations:

$$
\left.\begin{array}{ccc}
L_{f_{0}} V_{0}(x) & = & 0 \\
L_{f_{1}} V_{0}(x) & = & 0 \\
& \vdots & \\
L_{f_{m}} V_{0}(x)= & 0
\end{array}\right\} \Longrightarrow L_{f_{0}} L_{G} V_{0}(x)=-\sum_{i=1}^{L} \sum_{k=1}^{m} \frac{\left(L_{a d_{f_{0}}^{i}\left(f_{k}\right)} V_{0}(x)\right)^{2}}{\left(V_{0}\right)^{\alpha_{i, k}}}
$$

Considering that $\mathcal{W}_{L}\left(V_{0}\right)=\{0\}$, it follows that condition (22) is satisfied by the vector field $G$. 


\section{The method to Design control Lyapunov functions. Some extensions}

Theorems 4.2 and 4.3 give a method to design a strict Lyapunov function for homogeneous systems that satisfy Jurdjevic-Quinn conditions (Assumpt. 3.1) with a homogeneous function $V_{0}$ :

Step 1: Design a vector field $G$ that satisfies the condition (22). Theorem 4.3 gives a "universal" method to obtain it, but many other solutions $G$ for (22) may be considered. They produce different control Lyapunov functions, possibly simpler, possibly "better". See the examples and remark below.

Step 2: Compute $V_{\lambda}$ or $W_{\lambda}$ according to $(17,18)$, or (19). Formula (19) always yields explicit control Lyapunov functions for $\lambda$ positive and small enough. If it is possible to compute explicitly the flow of $G$, then one may also take $V_{\lambda}$ given by (17). Finally other formulae than (19) are possible to satisfy (18). See (49) for instance.

Step 3: Design a feedback law that makes $V_{\lambda}$ or $W_{\lambda}$ strictly decreasing. Equations (11) and (12) give a possibility to obtain it.

Remark 5.1. It is possible that a preliminary feedback is necessary to transform the system in a JurdjevicQuinn type system (see Rem. 3.2). Then, if we denote by $f_{0}$ the drift vector field for the original system, the assumptions are in fact met with $\widetilde{f}_{0}=f_{0}+\lambda_{1} f_{1}+\cdots+\lambda_{m} f_{m}$ instead of $f_{0}$. Of course, this preliminary feedback is in general necessary: there is no reason for $L_{\tilde{f}_{0}} V_{0} \leq 0$ to imply $L_{f_{0}} V_{0} \leq 0$, for instance. However, being a Control Lyapunov function is obviously feedback invariant: from the definition given by equation (2), a function $V$ is control Lyapunov function for the system defined by $\left(f_{0}, f_{1}, \ldots, f_{m}\right)$ if and only if it is a control Lyapunov function for the system defined by $\left(\widetilde{f}_{0}, f_{1}, \ldots, f_{m}\right)$. Hence our results yield control Lyapunov functions not only for the Jurdjevic-Quinn type system $\left(\tilde{f}_{0}, f_{1}, \ldots, f_{m}\right)$, but also for the original system. Consequently, in Step 3, the design of the feedback law can be made without taking into account the preliminary feedback. This feedback is used for constructing the CLF, but not in the control design itself, contrary to the Jurdjevic-Quinn design. See for instance the example in Section 7: formula (44) considers the original system (37) and does not take into account the preliminary feedback (38) that was necessary to design the control Lyapunov function $W_{\lambda}$.

Remark 5.2 (On the homogeneity requirement). The assumption of homogeneity is not very natural. It is a convenient case where we can restrict our attention to the situation on the homogeneous sphere, take advantage of the compactness, and extend the result to all the space. This condition is of course not necessary. The TORA example (see Sect. 8) is a good illustration of this: the model does not meet homogeneity hypothesis, but a control Lyapunov function is obtained by reshaping $V_{0}$ in $W_{\lambda}$ with a vector field $G$ that meets condition (22).

Remark 5.3 (On the choice of $G$ ). Formula $(26,27)$ gives a "universal" way to obtain a vector field $G$ that satisfies condition (22). However, this equation is very undetermined, and it may be a good idea to choose another solution than $(26,27)$.

It is easy to see that the form of equation (22) yields rather simple equations for the coefficients $\lambda_{i, k}$. Formula (27) has the advantage of singling out a solution basically obtained by taking zero for $\lambda_{i, k}$ with the highest $i$ (in (26) $i$ "should" naturally range from 0 to $L$ instead of $L-1$ ), but many other possibilities might be used. For instance the choices of the vector fields $G$ in sections 7 and 8 for the rigid body and the TORA systems, are obtained writing explicitely condition (22) and solving simple partial differentials equations.

\section{THE LINEAR CASE}

Let us make a short visit in the linear world. Consider the linear system given by:

$$
\dot{x}=A x+B u,
$$

with $x \in \mathbb{R}^{n}$ and $u \in \mathbb{R}^{m}$. 
For a quadratic function $V_{0}(x)=\frac{1}{2} x^{T} Q x$ (where $Q$ is a symmetric positive definite matrix), the equations of the set $\mathcal{W}_{L}\left(V_{0}\right)$ (see (13)) are given by:

$$
x^{T}\left(A^{T} Q+Q A\right) x=\left(A^{i} B\right)^{T} Q x=0, \quad i=0 \ldots n-1 .
$$

It is well-known that, from Cayley-Hamilton theorem, the equations for $i \geq n$ are redundant. Assume that the system (33) satisfies the Jurdjevic-Quinn conditions defined by Assumption 3.1:

(a) condition (14) becomes: $A^{T} Q+Q A \leq 0$,

(b) condition (15) becomes: $r k\left(A^{T} Q+Q A, B, A B, \ldots, A^{n-1} B\right)=n$.

Note that if $A^{T} Q+Q A$ is identically equal to zero then (a) is satisfied and (b) is equivalent to the controllability condition: $r k\left\{B, A B, \ldots, A^{n-1} B\right\}=n$.

From Proposition 3.3, the linear feedback $u=-B^{T} Q x$ makes the origin of the closed-loop system

$$
\dot{x}=\left(A-B B^{T} Q\right) x
$$

asymptotically stable, although $V_{0}$ is not assigned to be a Lyapunov function. For such systems the problem of finding a "strict" Lyapunov function can be solved via Lyapunov equation (see for instance [4]): for each symmetric positive definite matrix $M$ there exists one and only one positive definite matrix $Q_{1}$ satisfying the linear equation:

$$
\left(A-B B^{T} Q\right)^{T} Q_{1}+Q_{1}\left(A-B B^{T} Q\right)=-M .
$$

Our result gives an alternative method to tackle this problem, without solving this equation. Indeed, we may apply Theorem 4.3: formula (26) gives, by a simple computation:

$$
G(x)=\sum_{k=0}^{n-2} A^{k} B \sum_{i=0}^{n-k-2}(-1)^{i}\left(A^{(k+2 i+1)} B\right)^{T} Q x .
$$

Then Theorem 4.2 proves that $V_{\lambda}$ (resp. $W_{\lambda}$ ), defined by,

$$
V_{\lambda}=\frac{1}{2} x^{T} e^{\lambda G^{T}} Q e^{\lambda G} x \quad\left(\text { resp. } \quad W_{\lambda}=\frac{1}{2} x^{T}\left(I d+\lambda G^{T}\right) Q(I d+\lambda G) x\right)
$$

is a control Lyapunov function for $\lambda$ small enough.

Let us apply this method on the most simple example: a double integrator with a preliminary feedback that makes the system conservative:

$$
\left\{\begin{array}{l}
\dot{x}_{1}=x_{2}, \\
\dot{x}_{2}=-x_{1}+u .
\end{array}\right.
$$

This system satisfies the above conditions with $Q=I d$, i.e. $V_{0}=\frac{1}{2} x_{1}{ }^{2}+\frac{1}{2} x_{2}{ }^{2}$. Applying our method, we obtain $G$ via formula (35):

$$
G(x)=\left[\begin{array}{ll}
0 & 0 \\
1 & 0
\end{array}\right]\left[\begin{array}{l}
x_{1} \\
x_{2}
\end{array}\right] .
$$

Then a control Lyapunov function is obtained via formula (36), with in this case $e^{\lambda G}=I d+\lambda G$ :

$$
V_{\lambda}(x)=\frac{1}{2} x_{1}^{2}+\frac{1}{2}\left(x_{2}+\lambda x_{1}\right)^{2} .
$$


Since $\dot{V}_{\lambda}(x)=-\lambda x_{1}^{2}+\left(x_{2}+\lambda x_{1}\right)\left(u+\lambda x_{2}\right)$, the function $V_{\lambda}$ is a control Lyapunov function for all $\lambda>0$. Note that controlling the first equation by $x_{2}=-\lambda x_{1}$ and using classical methods to "add an integrator" yields the same Lyapunov function.

\section{Example: Control of the angular velocity of a Rigid Body}

The problem of stabilization of the velocity of a rigid body with two torques has been first solved in $[1,4]$. In [20], Morin gives a homogeneous control law that allows him to prove asymptotic stability together with robustness towards general errors on the model. These articles prove asymptotic stability using JurdjevicQuinn methods. In this example, a control Lyapunov function is obtained via our method.

Consider a rigid body in an inertial reference frame. We note $\omega_{1}, \omega_{2}$ and $\omega_{3}$ the angular velocity components along the principal axes of inertia. $j_{1}, j_{2}$ and $j_{3}$ denotes the principal moments of inertia. The Euler equations yield the following model:

$$
\left\{\begin{array}{l}
\dot{\omega}_{1}=c_{1} \omega_{2} \omega_{3}+\frac{\tau_{1}}{j_{1}} \\
\dot{\omega}_{2}=c_{2} \omega_{1} \omega_{3}+\frac{\tau_{2}}{j_{2}} \\
\dot{\omega}_{3}=c_{3} \omega_{1} \omega_{2}
\end{array}\right.
$$

with $c_{1}=\frac{j_{2}-j_{3}}{j_{1}}, c_{2}=\frac{j_{3}-j_{1}}{j_{2}}, c_{3}=\frac{j_{1}-j_{2}}{j_{3}}$.

A preliminary feedback is proposed in [20] to make the system satisfy the Jurdjevic-Quinn hypotheses.

$$
\left\{\begin{array}{l}
\tau_{1}=j_{1}\left(-c_{1} \omega_{2} \omega_{3}+\beta c_{3} \omega_{1} \omega_{2}+k_{1}\right) \\
\tau_{2}=j_{2}\left(-\left(c_{2}+\mu c_{3}\right) \omega_{1} \omega_{3}+k_{2}\right)
\end{array}\right.
$$

We obtain the new system:

$$
\begin{gathered}
\dot{\omega}=f_{0}(\omega)+k_{1} f_{1}+k_{2} f_{2} \\
f_{0}=\left[\begin{array}{r}
\beta c_{3} \omega_{1} \omega_{2} \\
-\mu c_{3} \omega_{1} \omega_{3} \\
c_{3} \omega_{1} \omega_{2}
\end{array}\right], f_{1}=\left[\begin{array}{l}
1 \\
0 \\
0
\end{array}\right], f_{2}=\left[\begin{array}{l}
0 \\
1 \\
0
\end{array}\right],
\end{gathered}
$$

where $k_{1}$ and $k_{2}$ are the new controls of the system. This system is of Jurdjevic-Quinn type with the positive definite and radially unbounded function $V_{0}$ defined by

$$
V_{0}=\frac{1}{2}\left(\omega_{1}-\beta \omega_{3}\right)^{2}+\frac{1}{2} \omega_{2}^{2}+\frac{\mu}{2} \omega_{3}^{2}
$$

In fact it is easy to verify that the set $\mathcal{W}_{1}\left(V_{0}\right)$ is reduced to $\{0\}$. The stabilizing control law given in [20] is obtained applying Proposition 3.3 and modifying (16) in order to keep the homogeneity of the closed-loop system:

$$
\left\{\begin{array}{l}
k_{1}=-\alpha_{1}\left|L_{f_{1}} V_{0}\right| L_{f_{1}} V_{0} \\
k_{2}=-\alpha_{2}\left|L_{f_{2}} V_{0}\right| L_{f_{2}} V_{0}
\end{array}\right.
$$

The system (39) is a Jurdjevic-Quinn type system with a non strict Lyapunov function $V_{0}$ which is homogeneous of degree 2 with respect to the standard dilation $\delta_{\mu}^{r}$ (with $r=(1,1,1)$ ). In addition the vector fields $f_{0}, f_{1}$ and $f_{2}$ are homogeneous of degree $1,-1$ and -1 . As a consequence, we are able to design a strict Lyapunov function (this is new to our knowledge). Details of calculations can be found in [8]. 


\section{Design of $G$}

One can use Theorem 4.3 to design the vector field $G$, but the following $\operatorname{simpler} G$ also satisfies condition (22) (left to the reader):

$$
G(\omega)=\left[\begin{array}{c}
0 \\
\frac{\beta \mu c_{3} \omega_{3}^{2}}{\left(2 V_{0}\right)^{\frac{1}{2}}} \\
0
\end{array}\right]
$$

\section{Design of the control Lyapunov function}

The flow of $G$ does not have a simple expression, so we use formula (19). It yields:

$$
W_{\lambda}=\frac{1}{2}\left(\omega_{1}-\beta \omega_{3}\right)^{2}+\frac{1}{2}\left(\omega_{2}+\lambda \frac{\beta \mu c_{3} \omega_{3}^{2}}{\sqrt{2 V_{0}}}\right)^{2}+\frac{\mu}{2} \omega_{3}^{2} .
$$

One can check that $W_{\lambda}$ is a control Lyapunov function if $0<\lambda<\frac{1}{\beta c_{3}}$.

\section{Design of the new control law}

Of course, $W_{\lambda}$ is not in general a Lyapunov function for the control law defined by (38) and (41). One has to design a new control law that assigns $W_{\lambda}$ to be decreasing. Moreover, as it is underlined in Remark 5.1, $W_{\lambda}$ is a control Lyapunov function for the original system. Then, equations (11) and (12) give the following expression:

$$
\left\{\begin{array}{l}
\tau_{1}=-\alpha\|\omega\|_{\delta^{r}} L_{f_{1}} W_{\lambda} \\
\tau_{2}=-\alpha\|\omega\|_{\delta^{r}} L_{f_{2}} W_{\lambda}
\end{array}\right.
$$

with $\alpha$ sufficiently large:

$$
\alpha>\sup _{\omega \in T} \frac{c_{1} \omega_{2} \omega_{3} \frac{\partial W_{\lambda}}{\partial x_{1}}(\omega)+c_{2} \omega_{1} \omega_{3} \frac{\partial W_{\lambda}}{\partial x_{2}}(\omega)+c_{3} \omega_{1} \omega_{2} \frac{\partial W_{\lambda}}{\partial x_{3}}(\omega)}{\left(\left(\frac{1}{j_{1}} \frac{\partial W_{\lambda}}{\partial x_{1}}(\omega)\right)^{2}+\left(\frac{1}{j_{2}} \frac{\partial W_{\lambda}}{\partial x_{2}}(\omega)\right)^{2}\right)\|\omega\|_{\delta^{r}}}
$$

where $T=\left\{\omega,\|\omega\|_{\delta^{r}}=1, c_{1} \omega_{2} \omega_{3} \frac{\partial W_{\lambda}}{\partial x_{1}}(\omega)+c_{2} \omega_{1} \omega_{3} \frac{\partial W_{\lambda}}{\partial x_{2}}(\omega)+c_{3} \omega_{1} \omega_{2} \frac{\partial W_{\lambda}}{\partial x_{3}}(\omega) \geq 0\right\}$.

\section{Robustness to misalignment of actuators}

There is no reason for these control laws to have better robustness or performance properties than these given in [20], but it gives a tool (the negative definite function $\dot{W}_{\lambda}$ ) to analyze it. Let us quantify, for instance, the tolerable errors on actuators misalignment with the principal axes. We consider the initial system (37):

$$
\dot{\omega}=X_{0}(\omega)+\tau_{1} f_{1}+\tau_{2} f_{2}
$$

with $\left(\tau_{1}, \tau_{2}\right)$ defined by the addition of the preliminary feedback and the control law we have made $\left(k_{1}, k_{2}\right)$.

Let $\left(\varphi_{1}, \theta_{1}\right)$ and $\left(\varphi_{2}, \theta_{2}\right)$ be angles between the position of the torques and the principal axes. That means the vector fields $f_{1}$ and $f_{2}$ of the system (45) are given by:

$$
f_{1}=\left[\begin{array}{c}
\cos \left(\varphi_{1}\right) \\
\sin \left(\varphi_{1}\right) \cos \left(\theta_{1}\right) \\
\sin \left(\varphi_{1}\right) \sin \left(\theta_{1}\right)
\end{array}\right] f_{2}=\left[\begin{array}{c}
\sin \left(\varphi_{2}\right) \cos \left(\theta_{2}\right) \\
\cos \left(\varphi_{2}\right) \\
\sin \left(\varphi_{2}\right) \sin \left(\theta_{2}\right)
\end{array}\right] .
$$

Then we have:

$$
\dot{W}_{\lambda}=L_{f_{0}} W_{\lambda}+k_{1} L_{\hat{f}_{1}} W_{\lambda}+k_{2} L_{\hat{f}_{2}} W_{\lambda}+\tau_{1} L_{f_{1}-\hat{f}_{1}} W_{\lambda}+\tau_{2} L_{f_{2}-\hat{f}_{2}} W_{\lambda} .
$$


We defined three positive constants $\beta_{1}, \beta_{2}$ and $\beta_{3}$ :

$$
\left\{\begin{array}{l}
\beta_{1}=-\sup _{\|\omega\|_{\delta^{r}}=1}\left(L_{f_{0}} W_{\lambda}+k_{1} L_{\hat{f}_{1}} W_{\lambda}+k_{2} L_{\hat{f}_{2}} W_{\lambda}\right) \\
\beta_{2}=\sup _{\|\omega\|_{\delta^{r}=1}} \tau_{1}\left\|\frac{\partial W_{\lambda}}{\partial \omega}\right\|_{\delta^{r}} \\
\beta_{3}=\sup _{\|\omega\|_{\delta^{r}=1}} \tau_{2}\left\|\frac{\partial W_{\lambda}}{\partial \omega}\right\|_{\delta^{r}}
\end{array}\right.
$$

Then we deduce:

$$
\dot{W}_{\lambda} \leq-\|\omega\|_{\delta^{r}}^{3}\left(\beta_{1}-2 \beta_{2}\left|\sin \left(\frac{\varphi_{1}}{2}\right)\right|-2 \beta_{3}\left|\sin \left(\frac{\varphi_{2}}{2}\right)\right|\right) .
$$

Consequently, if $\varphi_{1}$ and $\varphi_{2}$ are small enough to satisfy the following equation,

$$
2 \beta_{2}\left|\sin \left(\frac{\varphi_{1}}{2}\right)\right|+2 \beta_{3}\left|\sin \left(\frac{\varphi_{2}}{2}\right)\right|<\beta_{1}
$$

then the origin remains an asymptotically stable equilibrium.

\section{ExAmple: The TORA System}

Using our ideas to find some control Lyapunov function for this system was suggested by R. Sepulchre, from Université de Liège. The following is a summary of [10] (Sect. 4).

Let us consider the mechanical system called TORA (Translational Oscillator with Rotating Actuator) in [23] and RTAC (Rotational/Translational proof-mass Actuator) in [5,6,27]. It consists of a platform connected to a fixed frame of reference by a linear spring. The platform can oscillate without friction in the horizontal plane. On the platform, an eccentric rotating mass is actuated by a DC motor. The control of this rotating motion is used to dampen the translational oscillations of the platform. A precise description is given in [5].

$$
\begin{aligned}
\dot{x}_{1} & =x_{2} \\
\dot{x}_{2} & =\frac{-x_{1}+\varepsilon x_{4}^{2} \sin x_{3}}{1-\varepsilon^{2} \cos ^{2} x_{3}}+\frac{-\varepsilon \cos x_{3}}{1-\varepsilon^{2} \cos ^{2} x_{3}} u \\
\dot{x}_{3} & =x_{4} \\
\dot{x}_{4} & =\frac{\varepsilon \cos x_{3}\left(x_{1}-\varepsilon x_{4}^{2} \sin x_{3}\right)+u}{1-\varepsilon^{2} \cos ^{2} x_{3}}
\end{aligned}
$$

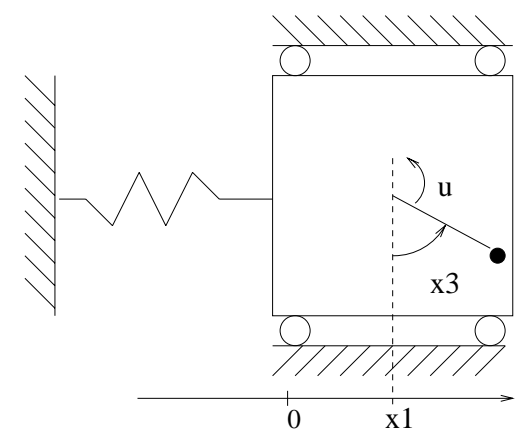

In [23], this system is extensively used as an illustrative example, and the papers [5,6,27] also propose various control methods. The following is an illustration of our methods on this example to derive a (global) CLF. We do not propose new or "better" control laws.

Setting the new state variables $z_{1}=x_{1}+\epsilon \sin x_{3}, z_{2}=x_{2}+\epsilon x_{4} \cos x_{3}, z_{3}=x_{3}, z_{4}=x_{4}$, a preliminary feedback (see $[10,23]$ for more details) yields the following control affine system:

$$
\dot{z}=f_{0}(z)+v f_{1}(z)
$$


where $f_{0}$ and $f_{1}$ are, in the coordinates $\left(z_{1}, z_{2}, z_{3}, z_{4}\right)$,

$$
f_{0}=\left[\begin{array}{c}
z_{2} \\
-z_{1}+\varepsilon \sin z_{3} \\
z_{4} \\
\varepsilon x_{1} \cos z_{3}-k_{1} z_{3}
\end{array}\right] \quad f_{1}=\left[\begin{array}{l}
0 \\
0 \\
0 \\
1
\end{array}\right]
$$

Let us define the following positive definite and radially unbounded function $V_{0}$ :

$$
V_{0}(z)=\frac{1}{2}\left(z_{1}-\varepsilon \sin z_{3}\right)^{2}+\frac{1}{2} z_{2}^{2}+\frac{k_{1}}{2} z_{3}^{2}+\frac{1}{2} z_{4}^{2}
$$

with $k_{1}$ some positive constant.

We have $L_{f_{0}} V_{0} \equiv 0$ and a computation of the $L_{f_{0}}^{j} L_{f_{1}} V_{0}$ for $j=1,2,3$, shows that this system satisfies the Jurdjevic-Quinn conditions (Assumpt. 3.1); precisely, $\mathcal{W}_{3}\left(V_{0}\right)=\{0\}$.

Unfortunately we can not actually apply our method because this system does not meet the homogeneity assumptions of our theorems. Yet in the following we look for a complete vector field $G$ satisfying condition (22), we give a function $W_{\lambda}$ that satisfies formula (18), and we prove that it is a control Lyapunov function for system (46).

The first step consists in finding a vector field $G$ that meets the condition (22). Instead of using formula $(26,27)$, we find another simpler vector field $G$ that satisfies this condition. Indeed, Let $G$ be defined by:

$$
G\left(x_{1}, z_{2}, z_{3}, z_{4}\right)=\left[\begin{array}{c}
-\varepsilon^{2} \rho\left(z_{2}\right) \cos ^{2} z_{3} \\
0 \\
2 \varepsilon \rho\left(z_{2}\right) \cos z_{3} \\
-\varepsilon x_{1}\left(1+2 \rho^{\prime}\left(z_{2}\right)\right) \cos z_{3}+k_{1} z_{3}-2 \varepsilon z_{4} \rho\left(z_{2}\right) \sin z_{3}
\end{array}\right]
$$

where $\rho$ is a smooth function of $z_{2}$ that is bounded, whose derivative is positive, bounded and such that:

$$
z_{2} \neq 0 \Rightarrow z_{2} \rho\left(z_{2}\right)>0
$$

A simple computation shows that this vector field satisfies condition (22) (see [10]). Following the idea of our method, we define $W_{\lambda}$. The following formula gives a $W_{\lambda}$ that differs from (19), but satisfies (18):

$$
\begin{aligned}
W_{\lambda}= & \frac{1}{2}\left(x_{1}-\lambda \varepsilon^{2} \rho\left(z_{2}\right) \cos ^{2} z_{3}\right)^{2}+\frac{1}{2} z_{2}{ }^{2}+\frac{k_{1}}{2}\left(z_{3}+2 \lambda \varepsilon \rho\left(z_{2}\right) \cos z_{3}\right)^{2} \\
& +\frac{1}{2}\left(\left(1-2 \lambda \varepsilon \rho\left(z_{2}\right) \sin z_{3}\right) z_{4}-\lambda \varepsilon x_{1}\left(1+2 \rho^{\prime}\left(z_{2}\right)\right) \cos z_{3}+\lambda k_{1} z_{3}\right)^{2} .
\end{aligned}
$$

Recall that $L_{f_{1}} W_{\lambda}$ is simply $\partial W_{\lambda} / \partial z_{4}$. Hence

$$
\begin{aligned}
L_{f_{1}} W_{\lambda} & =\left(1-2 \lambda \varepsilon \rho\left(z_{2}\right) \sin z_{3}\right)^{2} \xi_{4} \\
\text { with } \xi_{4} & =z_{4}+\lambda \frac{k_{1} z_{3}-\varepsilon x_{1}\left(1+2 \rho^{\prime}\left(z_{2}\right)\right) \cos z_{3}}{1-2 \lambda \varepsilon \rho\left(z_{2}\right) \sin z_{3}} .
\end{aligned}
$$

This vanishes either when $\xi_{4}=0$ or when $1-2 \lambda \varepsilon \rho\left(z_{2}\right) \sin z_{3}=0$. As $\rho$ is bounded we can take $\lambda$ such that $2 \lambda \varepsilon \rho\left(z_{2}\right)<1$. Provided this is satisfied, a careful computation yields, 


$$
\begin{aligned}
\dot{W}_{\lambda}= & -\lambda\left[k_{1} z_{3}-\varepsilon \cos z_{3}\left(x_{1}-\lambda\left(k_{1}+\frac{\varepsilon^{2}}{2} \cos ^{2} z_{3}\right) \rho\left(z_{2}\right)\right)\right]^{2} \\
& -\lambda \varepsilon^{2} \cos ^{2} z_{3} \rho^{\prime}\left(z_{2}\right)\left[x_{1}-\lambda \frac{\varepsilon^{2}}{2} \cos ^{2} z_{3} \rho\left(z_{2}\right)\right]^{2} \\
& -\lambda \varepsilon^{2} \cos ^{2} z_{3} \rho\left(z_{2}\right)\left[z_{2}-\lambda^{2} \rho\left(z_{2}\right)\left(\frac{\varepsilon^{4}}{4}\left(1+\rho^{\prime}\left(z_{2}\right)\right) \cos ^{4} z_{3}+k_{1} \varepsilon^{2} \cos ^{2} z_{3}+k_{1}^{2}\right)\right] \\
& +\xi_{4}\left[\lambda R\left(\lambda, \varepsilon, x_{1}, z_{2}, z_{3}, z_{4}\right)+k_{1} z_{3}-\varepsilon x_{1} \cos z_{3}\right. \\
& \left.\times \frac{\left(1-2 \lambda \varepsilon \rho\left(z_{2}\right) \sin z_{3}\right)^{2}}{1-\varepsilon^{2} \cos ^{2} z_{3}}\left(\varepsilon \cos z_{3}\left(x_{1}-\varepsilon z_{4}^{2} \sin z_{3}\right)+u\right)\right],
\end{aligned}
$$

where $R$ is some function, whose expression is somewhat lengthy but can easily be handled with a computer algebra system.

The three first terms depend only on $x_{1}, z_{2}, z_{3}$, and they are a negative definite function of these three variables since $\rho^{\prime}\left(z_{2}\right)$ is everywhere positive, and $\left|\rho\left(z_{2}\right)\right| \leq a\left|z_{2}\right|$. Note that the requirements on $\rho$ are met for instance with $\rho\left(z_{2}\right)=\frac{2}{\pi} \operatorname{Arctan} z_{2}$, and that, if $k_{1}=1$ and $\varepsilon=0.1, \lambda$ only has to be taken slightly less than 1 .

Then equation (52) clearly proves that $W_{\lambda}$ is a control Lyapunov function, and it satisfies the small control property since equation (52) allows one to derive very explicitly a continuous stabilizing control by making the last term non-positive, and negative when $\xi_{4} \neq 0$.

It is a pleasure to thank G. Sallet from INRIA Lorraine, Metz, for discussions on Jurdjevic-Quinn systems and for communicating [21,22], R. Sepulchre, from Université de Liège for many discussions and suggesting the example in Section 8, and L. Praly, from École des Mines, Paris, for fruitful comments and suggesting references [18, 19].

\section{REFERENCES}

[1] D. Aeyels, Stabilization of a class of nonlinear systems by smooth feedback control. Systems Control Lett. 5 (1985) $289-294$.

[2] Z. Artstein, Stabilization with relaxed control. Nonlinear Anal. TMA 7 (1983) 1163-1173.

[3] A. Bacciotti, Local stabilizability of nonlinear control systems. World Scientific, Singapore, River Edge, London, Ser. Adv. Math. Appl. Sci. 8 (1992).

[4] R.W. Brockett, Asymptotic stability and feedback stabilization, in Differential Geometric Control Theory, edited by R.W. Brockett, R.S. Millman and H.J. Sussmann. Basel-Boston, Birkäuser (1983) 181-191.

[5] R.T. Bupp, D.S. Bernstein and V.T. Coppola, A benchmark problem for nonlinear control design. Internat J. Robust Nonlinear Control 8 (1998) 307-310.

[6] — Experimental implementation of integrator back-stepping and passive nonlinear controllers on the RTAC testbed. Internat J. Robust Nonlinear Control 8 (1998) 435-457.

[7] J.-M. Coron, L. Praly and A.R. Teel, Feedback stabilization of nonlinear system: Sufficient conditions and lyapunov and input-output techniques, in Trends in Control, a European Perspective, edited by A. Isidori. Springer-Verlag (1995) 283-348.

[8] L. Faubourg, La déformation de fonctions de Lyapunov, Rapport de DEA d'automatique et informatique industrielle. INRIAUniversité de Lille 1 (1997).

[9] L. Faubourg and J.-B. Pomet, Strict control Lyapunov functions for homogeneous Jurdjevic-Quinn type systems, in Nonlinear Control Systems Design Symposium (NOLCOS'98), edited by H. Huijberts, H. Nijmeijer, A. van der Schaft and J. Scherpen. IFAC (1998) 823-829.

[10] L. Faubourg and J.-B. Pomet, Design of control Lyapunov functions for "Jurdjevic-Quinn" systems, in Stability and Stabilization of Nonlinear Systems, edited by D. Aeyels et al. Springer-Verlag, Lecture Notes in Contr. Es Inform. Sci. (1999) $137-150$.

[11] J.-P. Gauthier, Structure des Systèmes non-linéaires. Éditions du CNRS, Paris (1984).

[12] W. Hahn, Stability of Motion. Springer-Verlag, Berlin, New-York, Grundlehren Math. Wiss. 138 (1967).

[13] V. Jurdjevic and J.P. Quinn, Controllability and stability. J. Differential Equations 28 (1978) 381-389.

[14] M. Kawski, Homogeneous stabilizing feedback laws. Control Theory and Adv. Technol. 6 (1990), 497-516. 
[15] H.K. Khalil, Nonlinear Systems. MacMillan, New York, Toronto, Singapore (1992).

[16] J. Kurzweil, On the inversion of Ljapunov's second theorem on stability of motion. AMS Trans., Ser. II 24 (1956) 19-77.

[17] J.-P. LaSalle, Stability theory for ordinary differential equations. J. Differential Equations 4 (1968) 57-65.

[18] W. Liu, Y. Chitour and E. Sontag, Remarks on finite gain stabilizability of linear systems subject to input saturation, in $32^{\text {th }}$ IEEE Conf. on Decision and Control. San Antonio, USA (1993) 1808-1813.

[19] F. Mazenc, Stabilisation de trajectoires, ajout d'intégration, commandes saturées, Thèse de doctorat. École des Mines de Paris (1989).

[20] P. Morin, Robust stabilization of the angular velocity of a rigid body with two actuators. European J. Control 2 (1996) 51-56.

[21] R. Outbib and G. Sallet, Stabilizability of the angular velocity of a rigid body revisited. Systems Control Lett. 18 (1992) 93-98.

[22] G. Sallet, Historique des techniques de Jurdjevic-Quinn (private communication).

[23] R. Sépulchre, M. Janković and P.V. Kokotović, Constructive Nonlinear Control. Springer-Verlag, Comm. Control Engrg. Ser. (1997).

[24] E.D. Sontag, Feedback stabilization of nonlinear systems, in Robust control of linear systems and nonlinear control, Vol. 2 of proceedings of MTNS'89, edited by M.A. Kaashoek, J.H. van Schuppen and A. Ran. Basel-Boston, Birkhäuser (1990) 61-81.

[25] M. Spivak, A Comprehensive Introduction to Differential Geometry, Vol. 1. Publish or Perish, Houston, second Ed. (1979).

[26] J. Tsinias, Remarks on feedback stabilizability of homogeneous systems. Control Theory and Adv. Technol. 6 (1990) 533-542.

[27] J. Zhao and I. Kanellakopoulos, Flexible back-stepping design for tracking and disturbance attenuation. Internat J. Robust Nonlinear Control 8 (1998) 331-348. 